\title{
Near-Ground-State Cooling of Atoms Optically Trapped 300 nm Away from a Hot Surface
}

\author{
Y. Meng, ${ }^{1}$ A. Dareau, ${ }^{1}$ P. Schneeweiss, ${ }^{1, *}$ and A. Rauschenbeutel ${ }^{1,2, \dagger}$ \\ ${ }^{1}$ Vienna Center for Quantum Science and Technology, \\ TU Wien, Atominstitut, Stadionallee 2, 1020 Vienna, Austria \\ ${ }^{2}$ Department of Physics, Humboldt-Universität zu Berlin, 10099 Berlin, Germany
}

(Received 19 December 2017; revised manuscript received 21 May 2018; published 4 September 2018)

\begin{abstract}
Laser-cooled atoms coupled to nanophotonic structures constitute a powerful research platform for the exploration of new regimes of light-matter interaction. While the initialization of the atomic internal degrees of freedom in these systems has been achieved, a full preparation of the atomic quantum state also requires controlling the center-of-mass motion of the atoms at the quantum level. Obtaining such control is not straightforward because of the close vicinity of the atoms to the photonic system at ambient temperature. Here, we demonstrate cooling of individual neutral cesium atoms that are optically interfaced with light in an optical nanofiber, preparing them close to their three-dimensional motional ground state. The atoms are localized less than $300 \mathrm{~nm}$ away from the hot fiber surface. Ground-state preparation is achieved by performing degenerate Raman cooling, and the atomic temperature is inferred from the analysis of heterodyne fluorescence spectroscopy signals. Our cooling method can be implemented either with externally applied or guided light fields. Moreover, it relies on polarization gradients, which naturally occur for strongly confined guided optical fields. Thus, this method can be implemented in any trap based on nanophotonic structures. Our results provide an ideal starting point for the study of novel effects such as light-induced self-organization, the measurement of novel optical forces, and the investigation of heat transfer at the nanoscale using quantum probes.
\end{abstract}

DOI: 10.1103/PhysRevX.8.031054

Trapped laser-cooled atoms constitute a versatile experimental platform, with applications ranging from precision measurements [1,2], to quantum simulations of solid-state systems [3], to quantum communication [4]. A promising pathway is to interface cold atoms with light confined in nanophotonic structures, which offer a flexible design of the optical modes, very large coupling strengths, and enable the exploration of novel regimes of light-matter interaction [5]. Interfacing atoms with light guided in a nanophotonic element is typically achieved via the evanescent part of the optical mode. As these fields decay on the wavelength scale, emitters have to be very close to the surface of the photonic structure for purposes of reaching significant coupling strengths.

In order to harness the full potential of cold atoms and to build on their excellent coherence properties, the preparation of the atoms in a well-defined quantum-mechanical

\footnotetext{
philipp.schneeweiss@tuwien.ac.at

arno.rauschenbeutel@tuwien.ac.at
}

Published by the American Physical Society under the terms of the Creative Commons Attribution 4.0 International license. Further distribution of this work must maintain attribution to the author(s) and the published article's title, journal citation, and DOI.
Subject Areas: Atomic and Molecular Physics, Nanophysics, Quantum Physics state is required. The individual tasks of either trapping cold atoms close to nanophotonic structures [6,7] or quantum state preparation [8,9] of atoms confined in conventional, free-space traps have already been demonstrated. A quantum-level control of atoms in nanophotonic traps, however, constitutes a prime challenge as various effects, such as Johnson noise [10], patch potentials [11], or coupling to the phononic modes of the structure $[12,13]$, might lead to decoherence and heating. On a more general note, the very possibility of preparing quantum states including the external degrees of freedom (d.o.f.) of atoms close to a hot, macroscopic object cannot be taken for granted. In this regard, cooling atoms to the motional ground state constitutes a prerequisite for the preparation of more complex states. So far, only the cooling of a single d.o.f. has been shown close to a nanophotonic structure using microwave or Raman sideband cooling [14,15].

Here, we demonstrate three-dimensional cooling of individual cesium atoms that are located less than $300 \mathrm{~nm}$ away from the surface of a solid at ambient temperature, close to their motional ground state. The atoms are trapped near the nanofiber part of a tapered optical fiber using a nanofiber-based optical dipole trap [6]. We implement degenerate Raman cooling (DRC) [16-20], taking advantage of intrinsic trap properties. Compared to 
(a)

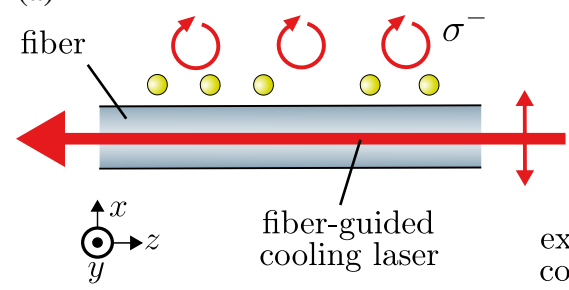

(b)

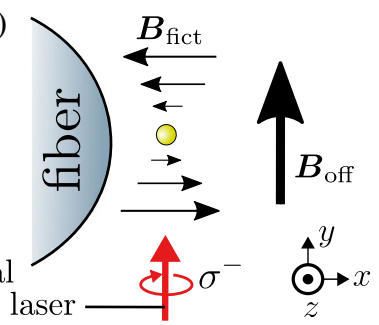

(c)

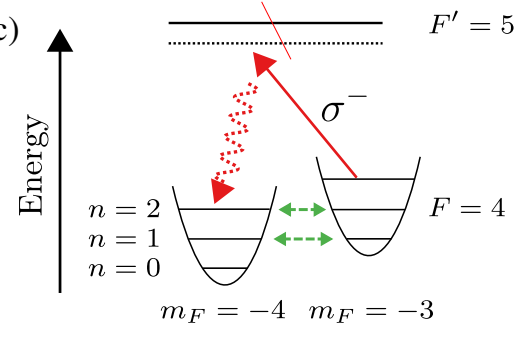

FIG. 1. (a) Schematic of laser-cooled cesium atoms (yellow spheres) interfaced with the evanescent part of a nanofiber-guided cooling light field (red arrows). The atoms are trapped in the $(x, z)$ plane. The polarization of the cooling light field at the position of the atoms is shown as red curved arrows. It is almost perfectly circular and corresponds to $\sigma^{-}$polarization [24]. (b) Illustration of the fictitious magnetic field $\boldsymbol{B}_{\text {fict }}$ induced by the blue-detuned trapping light field (black horizontal arrows) and the homogeneous external offset magnetic field $\boldsymbol{B}_{\text {off }}$ (vertical black arrow) for an atom located at the center of a nanofiber-based two-color trapping site. The external cooling laser, propagating along the $y$ axis, is indicated with a red arrow. (c) Schematic of degenerate Raman cooling (DRC). Each Zeeman substate $\left|m_{F}\right\rangle$, here shown for two states of the $F=4$ hyperfine manifold, has an associated set of motional states $|n\rangle$, here sketched for a simple 1D harmonic oscillator. The offset magnetic field $\boldsymbol{B}_{\text {off }}$ can be used to tune different states $\left|m_{F}, n\right\rangle$ and $\left|m_{F}^{\prime}, n^{\prime}\right\rangle$ $\left(m_{F} \neq m_{F}^{\prime}\right)$ into resonance. The coupling between these states (green dashed arrows) originates from the fictitious magnetic field $\boldsymbol{B}_{\text {fict }}$ gradient. To first order, only the state $\left|m_{F}=-4, n=0\right\rangle$ is not coupled by $\boldsymbol{B}_{\text {fict }}$. An additional laser field (illustrated as a red arrow) can be used to optically pump atoms into this state. Ideally, all atoms are then cooled down to the state $\left|m_{F}=-4, n=0\right\rangle$.

previous schemes [14,15], DRC is significantly less sensitive to magnetic field fluctuations, which otherwise may reduce the cooling efficiency. Moreover, spurious heating due to light scattering is less prominent for DRC. We can achieve cooling either using free-space light fields or with fiberguided light fields only. When cooling is applied continuously, the lifetime of atoms in the trap is increased by 1 order of magnitude and exceeds $1 \mathrm{~s}$, reaching the limit imposed by the background gas pressure in our setup. We use heterodyne fluorescence spectroscopy [21] in order to probe the atoms in the trap. This technique is robust with respect to magnetic field fluctuations for our settings and allows one to measure the atoms' temperature for all three motional d.o.f. This precise probing is key for the optimization of the cooling parameters and gives clear evidence that the atoms are cooled close to the motional ground state. Our work shows that full control of the three-dimensional motional state of the atoms at the level of a single quantum can be achieved despite the close vicinity of a hot, macroscopic body.

Our experiment relies on the evanescent part of far offresonant nanofiber-guided light fields to trap cesium atoms [6]. The trap is realized at the waist of a tapered optical fiber, where the fiber radius is reduced down to $250 \mathrm{~nm}$. Trapping is provided by a quasilinearly polarized [22] bluedetuned running wave with a free-space wavelength of $783 \mathrm{~nm}$ and a power of $17.8 \mathrm{~mW}$, and an orthogonally polarized red-detuned standing wave at a wavelength of $1064 \mathrm{~nm}$ with a total power of $2.88 \mathrm{~mW}$. The atoms are loaded into two diametric one-dimensional arrays of trapping sites, where they are confined in all three spatial dimensions. An $a b$ initio calculation of the trapping potential yields frequencies of $\left\{\omega_{x}, \omega_{y}, \omega_{z}\right\} / 2 \pi=$ $\{136,83,215\} \mathrm{kHz}$ and a trap minimum located about $280 \mathrm{~nm}$ away from the surface of the nanofiber. The calculated trap frequencies correspond to Lamb-Dicke parameters of $\left\{\eta_{x}, \eta_{y}, \eta_{z}\right\}=\{0.12,0.16,0.10\}$ for the $D_{2}$ line of cesium; i.e., the experiment operates in the LambDicke regime for atoms close to the ground state.

Because of the strong transverse confinement of the trapping light fields in the nanofiber, the atoms experience a strongly spatially varying vector ac Stark shift [14], also known as a fictitious magnetic field [23]. When the polarizations of the red-detuned guided light fields generating the standing wave are aligned, they do not generate a fictitious magnetic field, and only the contribution of the blue-detuned light field remains. Near the trap minimum, the resulting fictitious magnetic field $\boldsymbol{B}_{\text {fict }}$ dominantly points along the $x$ axis, and its magnitude varies linearly along $y$. Furthermore, since the fictitious magnetic field originates from the evanescent trapping light fields, its amplitude decays exponentially in the radial direction $(x)$. Hence, we model $\boldsymbol{B}_{\text {fict }}$ using the following formula:

$$
\boldsymbol{B}_{\text {fict }} \approx b y e^{-x / l} \boldsymbol{e}_{x}
$$

with $\boldsymbol{e}_{x}$ the unit vector along $x, b=1.6 \mathrm{G} / \mu \mathrm{m}$ and $l=$ $70 \mathrm{~nm}$ for our trap configuration. Near the trap minimum $(x, y=0)$, to first order, we can neglect the effect of the radial decay of the field, and we are left with $\boldsymbol{B}_{\text {fict }} \approx b y \boldsymbol{e}_{x}$, as illustrated in Fig. 1(b). The evolution of the spin and $y$ motional d.o.f. for an atom in the trap is then governed by the following Hamiltonian:

$$
\hat{H}=\hbar \omega_{y} \hat{a}_{y}^{\dagger} \hat{a}_{y}+g_{F} \mu_{B} \hat{\boldsymbol{F}} \cdot\left(\boldsymbol{B}_{\text {off }}+\boldsymbol{B}_{\text {fict }}\right),
$$

where $\hat{\boldsymbol{F}}$ is the total angular momentum operator, $g_{F}$ is the hyperfine Landé factor, and $\mu_{B}$ is the Bohr magneton. We have assumed the trap to be harmonic and introduced the corresponding annihilation operator $\hat{a}_{y}$. An additional homogeneous offset magnetic field, $\boldsymbol{B}_{\text {off }}=B_{\text {off }} \boldsymbol{e}_{y}$, points 
along the $y$ axis; see Fig. 1(b). Using our expression for $\boldsymbol{B}_{\text {fict }}$, the Hamiltonian (2) can be rewritten as

$\hat{H} / \hbar=\omega_{y} \hat{a}_{y}^{\dagger} \hat{a}_{y}+\Delta_{\text {off }} \hat{F}_{y}+\frac{\Omega}{2}\left(\hat{a}_{y}+\hat{a}_{y}^{\dagger}\right)\left(\hat{F}_{+}+\hat{F}_{-}\right)$,

where we have introduced $\hat{F}_{+}\left(\hat{F}_{-}\right)$as the spin raising (lowering) operator for the $\hat{F}_{y}$ eigenstates $\left(\hat{F}_{x}=\right.$ $\left.\left[\hat{F}_{+}+\hat{F}_{-}\right] / 2\right)$ and used $\hat{y}=y_{0}\left(\hat{a}_{y}+\hat{a}_{y}^{\dagger}\right)$. The second term in Eq. (3) corresponds to the Zeeman shift induced by $\boldsymbol{B}_{\text {off }}$ $\left(\Delta_{\text {off }} \propto B_{\text {off }}\right)$. The last term comes from the fictitious magnetic field gradient and induces a coherent coupling between the spin and motional d.o.f., equivalent to a Raman coupling. The spin-motion coupling strength is given by $\hbar \Omega=g_{F} \mu_{B} b y_{0}$ and effectively corresponds to the Zeeman shift induced by the fictitious magnetic field gradient $b$ over the size of the harmonic oscillator ground state $y_{0}$ along the $y$ axis. For our trap configuration, we find $\Omega=2 \pi \times 12 \mathrm{kHz}$. In the following, the eigenstates of $\hat{H}$ in the absence of spin-motion coupling $(\Omega=0)$ are denoted as $\left|m_{F}, n\right\rangle$, where $m_{F}$ is the projection of the hyperfine atomic spin along $y$; i.e., the magnetic quantum number is specified assuming a $y$-quantization axis. The motional state of the atom in the trap is labeled by $n$.

Hamiltonian (3) enables degenerate Raman cooling, as illustrated in Fig. 1(c). The offset magnetic field is tuned so that the energies of the states $\left|m_{F}=-4, n\right\rangle$ and $\mid m_{F}=$ $-3, n-1\rangle$ are degenerate. These two states are then resonantly coupled by the spin-motion coupling term, which removes (adds) one quantum of motional energy as the atom precesses to a higher (lower) Zeeman state. To obtain cooling, we continuously apply a $\sigma^{-}$-polarized light field to pump atoms back to the lower Zeeman state. In the Lamb-Dicke regime, the optical pumping preserves the motional state, and the atom is pumped to $\left|m_{F}=-4, n-1\right\rangle$. While this process continues, atoms accumulate in $\left|m_{F}=-4, n=0\right\rangle$ since this state is not resonantly coupled to any other state through spin-motion coupling.

According to this model, the cooling rate depends on the spin-motion coupling rate $\Omega$ and on the optical pumping rate $R_{\mathrm{op}}$ from $\left|m_{F}=-3\right\rangle$ to $\left|m_{F}=-4\right\rangle$, which have to be compared to decoherence and heating rates. We perform DRC on the $F=4 \rightarrow F^{\prime}=5$ transition of the $D_{2}$ line of cesium. Taking into account the relevant Clebsch-Gordan coefficients, we get $R_{\text {op }}=(4 / 5)(1 / 5) R_{\mathrm{sc}}$, where $R_{\mathrm{sc}}$ is the scattering rate for the cycling transition $\left(F=4, m_{F}=-4\right) \leftrightarrow$ $\left(F^{\prime}=5, m_{F^{\prime}}=-5\right)$, which can be estimated from the laser detuning and intensity. For typical experimental settings, we get $R_{\mathrm{sc}} \approx 40-120 \mathrm{~ms}^{-1}$, corresponding to $R_{\mathrm{op}} \approx 6-20 \mathrm{~ms}^{-1}$. From previous characterizations, we expect a heating rate of less than 1 motional quantum per millisecond in our setup [14,25], i.e, 1 order of magnitude smaller than the calculated values for $\Omega$ and $\gamma_{\mathrm{op}}$. The measured heating rate is about 3 orders of magnitude larger than the one observed in free-space optical microtraps [26]. This makes ground-state cooling in nanofiber-based traps challenging. Preliminary studies suggest that the heating in the nanofiber system is dominated by the optomechanical coupling of the trapping light fields to thermally excited longitudinal and flexural mechanical modes of the nanofiber.

This model for DRC can be extended by carrying out the Taylor series expansion of $\boldsymbol{B}_{\text {fict }}$, as given in Eq. (1), to higher orders in $x$. For instance, the next order yields a $x y$ term, which gives rise to a term $\hbar \Omega_{x y}\left(\hat{a}_{y}+\hat{a}_{y}^{\dagger}\right)\left(\hat{a}_{x}+\hat{a}_{x}^{\dagger}\right) \hat{F}_{x}$ in the Hamiltonian. This enables a simultaneous transfer of energy from the $x$ and $y$ motional d.o.f. to the spin d.o.f., as well as cooling along the $x$ axis. For our trap configuration, we expect $\Omega_{x y}=2 \pi \times 2.9 \mathrm{kHz}$. In general, the fictitious magnetic field in our setup is expected to show gradients in all spatial dimensions around the trap minimum. Along the $x$ and the $y$ directions, gradients are expected even in the ideal configuration [see Ref. [14] for an exact representation of $\boldsymbol{B}_{\text {fict }}$ in the $(x, y)$ plane]. A gradient along $z$ can arise due to residual misalignment of the polarizations of the two red-detuned trapping light fields. These gradients are responsible for coupling terms that enable DRC of all motional d.o.f.

In a typical experimental sequence, atoms are loaded in the nanofiber-based optical trap directly from a magnetooptical trap (MOT). A first stage of polarization gradient cooling (PGC) is performed in the trap, and the initial atom number $N_{0}$ is inferred from the absorption of a weak nanofiber-guided light field on the cycling transition of the $D_{2}$ line. The number of atoms remaining in the trap, $N_{\mathrm{at}}$, is measured by the same means at the end of the sequence. In order to perform DRC, we apply a $\sigma^{-}$-polarized laser field, hereafter denoted as the cooling laser. This laser can either propagate in free space or be guided in the nanofiber. In the former case, we use a laser field propagating along the $+y$ direction; see Fig. 1(b). This external laser field has a $1 / e^{2}$ diameter of $1.4 \mathrm{~mm}$, which is large enough to cover the full atomic sample. In the latter case, a quasilinearly polarized fiber-guided laser field is used, whose polarization axis lies in the plane of the atoms; see Fig. 1(a). Because of the strong transverse confinement of the light field in the nanofiber, the local polarization is then almost perfectly circular at the position of the atoms [24]. On one side of the nanofiber, the light field is $\sigma^{-}$polarized, thus enabling cooling. The light field is $\sigma^{+}$polarized on the other side, which results in heating and, eventually, in the loss of the atoms. Applying DRC with fiber-guided light thus allows us to selectively cool the atoms trapped on one side of the fiber while removing the atoms trapped on the other side.

A first signature of cooling is obtained by measuring the lifetime of the atoms in the trap; see inset of Fig. 2. In this experiment, the fraction of atoms left in the trap, $N_{\text {at }} / N_{0}$, 


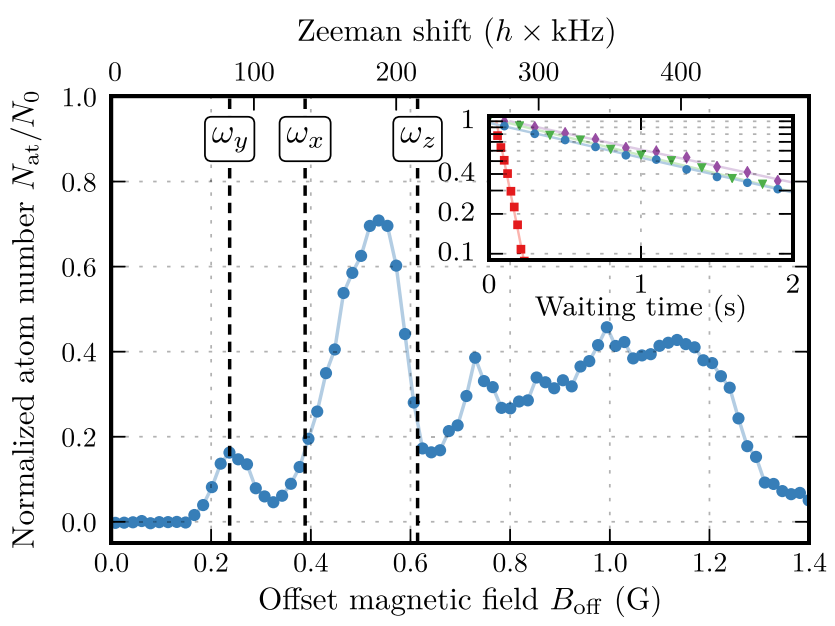

FIG. 2. Inset: Normalized number of trapped atoms measured after a variable time, in the absence of cooling (red squares), with DRC cooling using an external (blue circles) or a nanofiberguided (purple diamonds) laser field and with PGC using the MOT laser fields (green triangles). The measurements with DRC are performed at an offset magnetic field $B_{\text {off }}=0.5 \mathrm{G}$, with a peak intensity of $I_{0}=4.1 I_{\text {sat }}$ and a detuning of $-12 \Gamma$ for the external cooling laser. Here, $I_{\text {sat }}$ and $\Gamma$ are the saturation intensity and natural linewidth of the $D_{2}$ transition of cesium. The measurement using a nanofiber-guided laser field was performed with a peak intensity of $I_{0}=0.2 I_{\text {sat }}$ at the position of the atoms and a detuning of $-6.5 \Gamma$. The solid lines correspond to fits assuming an exponential decay, yielding a reference lifetime of 75(1) $\mathrm{ms}$ in the absence of cooling. In the presence of DRC, we observe lifetimes of 1650(20) ms and $1750(30) \mathrm{ms}$, respectively, with an external and a fiber-guided cooling laser. For PGC, we measure a lifetime of 1560(20) ms. Main graph: Normalized number of atoms still trapped after $500 \mathrm{~ms}$ of DRC, using a nanofiber-guided cooling laser, as a function of $B_{\text {off }}$. This measurement was performed with a peak intensity of $I_{0}=5.6 \times 10^{-2} I_{\text {sat }}$ at the position of the atoms and a detuning of $-3.6 \Gamma$. The corresponding energy shift between two adjacent Zeeman states is given at the top of the figure. Pronounced local maxima of the number of remaining atoms are discernible. We attribute these to resonances in the coupling between the motional and spin d.o.f., at which the cooling rate is maximized and, thus, the heating of atoms out of the trap efficiently counteracted. A vertical black dashed line indicates the position of the resonance expected from Eq. (3), using our $a b$ initio calculation of $\omega_{y}$. For completeness, the theoretical positions of resonances corresponding to the exchange of 1 motional quantum for other trap axes are also indicated.

is measured after a variable waiting time. Without any cooling (red squares), we observe an exponential decay with a $1 / e$ time constant of $\tau_{\text {ref }}=75(1) \mathrm{ms}$. When DRC is constantly applied using an external cooling laser (blue circles), the atoms are kept in the trap significantly longer $\left[\tau_{\mathrm{DRC}}^{\mathrm{ext}}=1650(20) \mathrm{ms}\right]$, reaching the regime in which collisions with the background gas in the vacuum chamber become the limiting factor. This conclusion is supported by an additional measurement (green triangles), in which we constantly apply PGC, which yields a lifetime in reasonable agreement with the DRC value $\left[\tau_{\mathrm{PGC}}=1560(20) \mathrm{ms}\right]$. A similar increase of the lifetime is observed when using a fiber-guided cooling laser to perform DRC [purple diamonds, $\left.\tau_{\mathrm{DRC}}^{\text {guided }}=1750(30) \mathrm{ms}\right]$. The large improvement of the trap lifetime observed in the presence of DRC indicates that this technique can effectively counteract heating mechanisms in the trap. Moreover, we know from previous work [14] that cooling a single d.o.f., namely, the $y$ d.o.f., is not sufficient to improve the trap lifetime. This indicates that a possible coupling of different d.o.f., e.g., due to the nonseparability of the trapping potential, is too weak to explain the observed increase of the lifetime. The increased lifetime is thus a first hint that all three motional d.o.f. are directly cooled with our method.

We investigate the coupling between the spin and the motional d.o.f. by performing DRC for different offset magnetic field strengths (see Fig. 2, main panel). Specifically, we record the number of atoms in the trap after $500 \mathrm{~ms}$ of DRC for different values of $B_{\text {off }}$. We observe pronounced local maxima, corresponding to the tuning of different motional states of adjacent spin states into resonance. A resonant coupling leads to an increased cooling rate, which results in a longer lifetime and thus in a larger number of atoms detected. The first local maximum, at $B_{\text {off }} \approx 0.25 \mathrm{G}$, corresponds to a resonant exchange of one spin excitation and one excitation of the azimuthal $(y)$ motional d.o.f., as predicted from the simple model illustrated in Fig. 1. The expected position of this resonance, deduced from the $a b$ initio calculation of $\omega_{y}$, is indicated by a vertical dashed line in Fig. 2. Other maxima are related to higher-order spin-motional coupling terms involving different motional d.o.f. Note that efficient cooling of the different d.o.f. is expected for different values of the offset magnetic field and that the optimum lifetime results from reaching a trade-off between the individual cooling efficiencies of all d.o.f. Moreover, the trap anharmonicity results in a temperature-dependent resonance condition. As a consequence of these two points, the accurate prediction of the position of these higher-order maxima is difficult.

Careful tuning of the cooling laser parameters is required to get efficient DRC. In the main panel of Fig. 3, we show the number of atoms in the trap after $80 \mathrm{~ms}$ of DRC as a function of the laser detuning and for three different laser powers. The measurement is performed with an offset magnetic field of $0.5 \mathrm{G}$, using the external cooling laser. The fraction of atoms left in the absence of DRC is indicated, for reference, with a horizontal dashed black line. A finer scan of the cooling laser power for a fixed detuning is shown in the inset of Fig. 3. We can clearly identify two limiting regimes. For low powers and/or large detunings, the scattering rate is reduced and so is the cooling efficiency, while small detunings and/or high powers lead to an increased recoil heating rate, which counteracts the cooling and even leads to significant atom losses. 


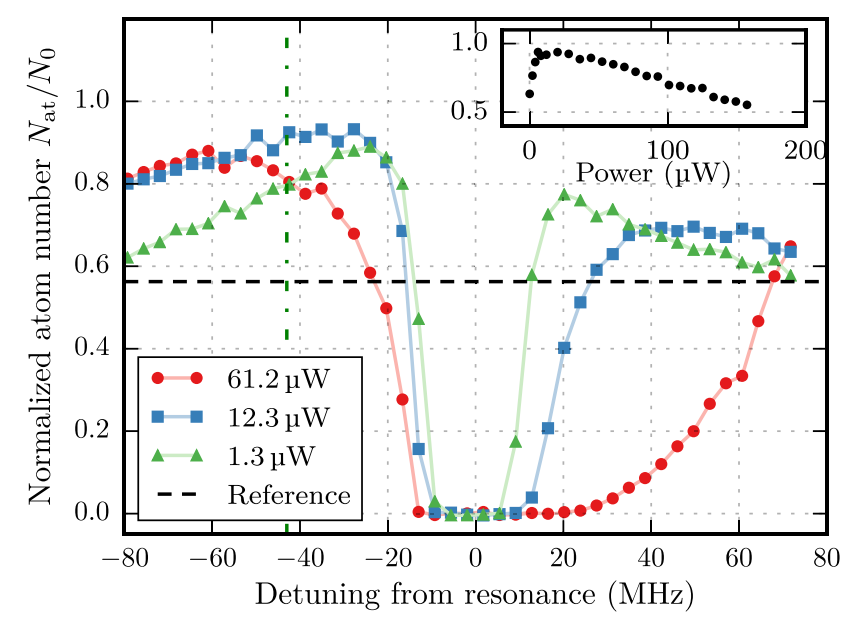

FIG. 3. Main graph: Normalized atom number remaining in the trap after $80 \mathrm{~ms}$ of DRC for various detunings of the external cooling laser field. Negative (positive) detuning values correspond to a red (blue) detuning of the laser field with respect to the $\left(6 S_{1 / 2}, F=4\right) \rightarrow\left(6 P_{3 / 2}, F^{\prime}=5\right)$ optical transition of the trapped cesium atoms. The measurement is repeated for different powers of the external cooling laser, $P_{0}=61.2 \mu \mathrm{W}$ (red circles), $12.3 \mu \mathrm{W}$ (blue squares), and $1.3 \mu \mathrm{W}$ (green triangles), corresponding to a peak intensity of $I_{0} / I_{\text {sat }}=6.8,1.4$, and 0.14 , respectively. The black dashed line indicates the fraction of atoms remaining in the trap in the absence of DRC. When the laser is too close to resonance, the cooling is outweighed by recoil heating, which induces atom losses. Moreover, the signal is asymmetric in the laser detuning, see main text. Inset: Number of remaining atoms in the presence of DRC as a function of the cooling laser power. The measurement is taken after $80 \mathrm{~ms}$ of DRC and at a detuning of $-9.4 \Gamma$ (vertical line in main graph). The signal is maximized for a power of about $15 \mu \mathrm{W}$, corresponding to $I_{0}=1.7 I_{\text {sat }}$.

(a)

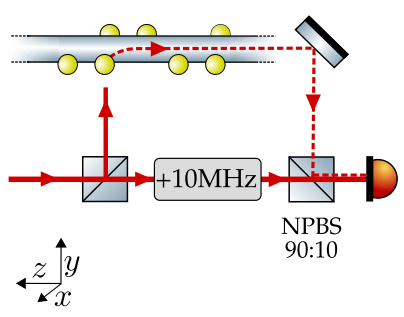

(b)

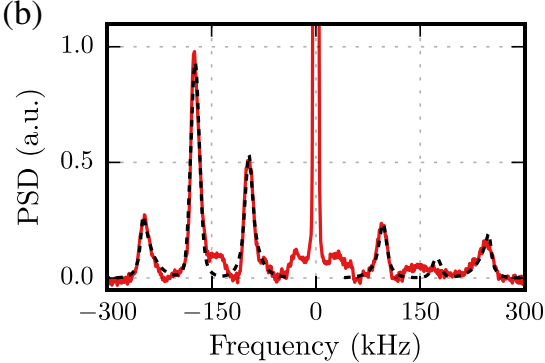

In addition to changing the scattering rate, scanning the power and detuning modifies the Zeeman-state-dependent ac Stark shift induced by the cooling laser itself, which either increases or reduces the shift induced by the offset magnetic field. The sign of the resulting effective Zeeman shifts depends on the laser detuning, and, close to resonance, their magnitude can be comparable to the energy level spacing in the trap. This can significantly alter the DRC resonance condition, and it explains the asymmetry of the cooling efficiency for positive and negative detuning in Fig. 3.

More information on the cooling can be obtained by analyzing the light scattered by the atoms. We measure the fluorescence spectrum while performing DRC with the external cooling laser using a heterodyne detection scheme $[21,27,28]$. During the DRC process, the atoms scatter light into the nanofiber. This light is guided to a beam splitter where it is combined with a reference laser field, derived from the cooling laser and frequency shifted by $10 \mathrm{MHz}$; see Fig. 4(a). The resulting beat note is recorded using a single photon counting module (SPCM). With this detector, we can also monitor the number of trapped atoms by measuring transmission spectra through the nanofiber at the beginning and the end of each experimental sequence. The motion of the atoms in the trap modulates the scattered light and gives rise to sidebands at the trap frequencies in the signal's power spectral density (PSD); see Figs. 4(b) and 4(c). In the azimuthal $(y)$ and axial $(z)$ directions, the sidebands arise from the phase modulation of, respectively, the incoming and the scattered photons. In the radial direction $(r)$, we expect an amplitude modulation for photons scattered in the evanescent part of the nanofiber-guided light field, which also results in a motional sideband. In the weak excitation limit, the scattering

FIG. 4. Measurement of trap frequencies and mean numbers of motional quanta of nanofiber-trapped atoms using heterodyne fluorescence spectroscopy (FS). (a) FS setup: The external cooling laser is propagating along the $+y$ direction and impinges on the atoms that are trapped in the $(x, z)$ plane (see also Fig. 1). During the DRC process, the atoms scatter a fraction of the light into the nanofiber. The light is guided to a 90:10 nonpolarizing beam splitter where it is combined with a reference laser field that is derived from the cooling laser and frequency shifted by $10 \mathrm{MHz}$. The resulting beat note is recorded using a SPCM. (b,c) PSD of the SPCM signal for an offset magnetic field of (b) $0.30 \mathrm{G}$ and (c) $0.51 \mathrm{G}$. The frequency axis is defined relative to the central beat note. During a single experimental cycle, the atoms are illuminated for $200 \mathrm{~ms}$ with a peak intensity of $I \approx 14 I_{\text {sat }}$ and a detuning of $-12 \Gamma$. For each realization, the PSD is obtained from a windowed Fourier transform of the SPCM signal (Welch method, 1-ms window). Using a SPCM in the time-tagging mode allows us to run this software-based data analysis a posteriori on the saved SPCM data. Spectra (b) and (c) are then obtained by averaging the resulting PSDs over around 6000 realizations. The dotted black line corresponds to a fit of the experimental data; see main text. From this fit, we extract mean numbers of excitations of (b) $\left\{\left\langle n_{x}\right\rangle,\left\langle n_{y}\right\rangle,\left\langle n_{z}\right\rangle\right\}=\{0.10(1), 0.78(5), 2.5(3)\}$ and (c) $\left\{\left\langle n_{x}\right\rangle,\left\langle n_{y}\right\rangle,\left\langle n_{z}\right\rangle\right\}=\{1.4(2), 0.58(2), 0.22(3)\}$, indicating that all motional d.o.f. can be cooled close to the ground state. 
process is mostly coherent. The width of the sidebands then depends on the cooling rate, the anharmonicity, and the inhomogeneity of the trapping potential. The temperature of the atomic ensemble can be inferred from the ratio of the amplitudes of the sidebands [29]. For a harmonic oscillator in the Lamb-Dicke regime, the mean number of excitations along $i \in\{x, y, z\}$ is given by $\left\langle n_{i}\right\rangle=S_{i}^{-} /\left(S_{i}^{+}-S_{i}^{-}\right)$, where $S_{i}^{ \pm}$is the amplitude of the sideband corresponding to the transition $n_{i} \rightarrow n_{i} \pm 1$. Since this thermometry method relies on analyzing the light scattered by the atoms during the cooling process, it only grants access to the temperature in the presence of DRC. Moreover, the time needed to obtain a sufficient signal-to-noise ratio, and thus a reliable temperature measurement, is longer than the typical cooling timescale. As a consequence, we can only perform steady-state temperature measurements. Our method is therefore not suited to perform time-resolved measurements of the temperature, which would be required in order to study, e.g., heating or cooling rates or to measure the temperature that results from other cooling methods, such as PGC.

In order to derive quantitative information from the recorded spectra, we perform a fit on experimental data. The shape of the sidebands is obtained by calculating the rate $\Gamma_{n_{i}, n_{i}^{\prime}}$ of each individual scattering process $n_{i} \rightarrow n_{i}^{\prime}$ using second-order perturbation theory [30,31]. Such a scattering event gives rise to a spectral contribution, whose amplitude and width depend on $\Gamma_{n_{i}, n_{i}^{\prime}}$. It is centered around a frequency $f_{n_{i}, n_{i}^{\prime}}=\Delta E_{n_{i}, n_{i}^{\prime}} / h$, where $\Delta E_{n_{i}, n_{i}^{\prime}}$ is the energy difference between the initial and final states, taking into account the trap anharmonicity. All the contributions are then added incoherently to obtain the spectrum. We assume a thermal distribution of the atoms in the trap. We then use the mean number of excitations $\left\{\left\langle n_{i}\right\rangle\right\}_{i=x, y, z}$ and the trap frequencies $\left\{\omega_{i}\right\}_{i=x, y, z}$ as fit parameters, together with a global scattering rate (setting the minimum width for the sidebands), an offset, and a global amplitude accounting for the combined detection efficiency.

A fit on the spectrum shown in Fig. 4(c) yields trap frequencies $\left\{\omega_{x}, \omega_{y}, \omega_{z}\right\} / 2 \pi=\{154,94,233\} \mathrm{kHz}$, which are in reasonable agreement with our ab initio calculation. The fitted sideband widths are on the order of 10 kilohertz, which sets an upper limit of about $10 \%$ for the inhomogeneity of the trapping frequencies in different sites along the nanofiber. The clear amplitude asymmetry of the Stokes and anti-Stokes sidebands in Figs. 4(b) and 4(c) is a signature of significant ground-state occupations. For the spectrum shown in Fig. 4(b), corresponding to an offset magnetic field of $B_{\text {off }}=0.30 \mathrm{G}$, our fit yields $\left\{\left\langle n_{x}\right\rangle,\left\langle n_{y}\right\rangle,\left\langle n_{z}\right\rangle\right\}=\{0.10(1), 0.78(5), 2.5(3)\}$, which corresponds to ground-state occupations of $91 \%$ and $56 \%$, respectively, for the radial $(x)$ and azimuthal $(y)$ motional states. The axial $(z)$ motion can be more efficiently cooled by changing the offset magnetic field. A spectrum recorded at $B_{\text {off }}=0.51 \mathrm{G}$ [Fig. 4(c)] indicates a mean number of axial excitations of $\left\langle n_{z}\right\rangle=0.22(3)$, corresponding to a ground-state occupation of $82 \%$. For the other motional d.o.f., we then find $\left\{\left\langle n_{x}\right\rangle,\left\langle n_{y}\right\rangle\right\}=\{1.4(2), 0.58(2)\}$. These temperature measurements were performed for settings that enable simultaneous cooling of multiple d.o.f., rather than minimizing the temperature of a single d.o.f.

We now discuss the mechanisms that can limit the final temperatures reached with our DRC scheme. In the idealized case without heating and neglecting off-resonant excitation, all atoms would end up in the motional ground state after a time that only depends on the cooling rate. With heating, the final temperature, and thus the mean number of motional quanta, is set by a competition between the cooling rate of atoms that have not yet reached the ground state and the rate with which atoms leave the ground state. The cooling rate depends both on the settings of the cooling laser (see Fig. 3) and on the amplitude of the offset magnetic field (see Fig. 2). Concerning heating, intrinsic fluctuations of the position and/or stiffness of the trap can never be fully avoided in the experiment. This gives rise to a background heating, whose rate was measured to be about 0.3 quanta/ms in the azimuthal $(y)$ direction [14]. Moreover, in contrast with many other implementations of DRC, the cooling laser in our experiment drives an optical cycling transition, $\left(6 S_{1 / 2}, F=4\right) \rightarrow$ $\left(6 P_{3 / 2}, F^{\prime}=5\right)$. In this case, the final state in the cooling process, $\left|m_{F}=-4, n=0\right\rangle$, is not a dark state; i.e., it is not decoupled from the laser. We choose this setting since our temperature measurement technique relies on the analysis of light scattered by the atoms. However, this scattering is a source of additional heating due to the transfer of photon recoil to the atoms. In particular, the intensity of the cooling laser field for the measurements in Fig. 4 is higher than the optimum intensity indicated in the inset of Fig. 3. We choose a larger intensity in order to increase the number of fluorescence photons collected and, hence, the signal-to-noise ratio in our spectra. Because of the increased recoil heating in this setting, we are convinced that the measured mean numbers of motional quanta constitute upper bounds of what can be achieved with the DRC method, e.g., when cooling is performed using a light field on a noncycling transition. We confirm with additional measurements that, in this case, we obtain comparable boosts in the lifetime of atoms in the trap, as shown in the inset in Fig. 2.

In summary, we have shown that degenerate Raman cooling can be efficiently implemented in nanofiber-based optical traps. Remarkably, this technique only requires one additional laser field, which can be fiber guided and which provides cooling for all three motional d.o.f. Cooling is enabled by the strong gradients of fictitious magnetic fields, which naturally arise when trapping atoms in evanescent fields [14]. This scheme is thus directly applicable to a vast variety of optical microtraps and, in particular, to traps based on other nanophotonic structures. Using a heterodyne 
fluorescence spectroscopy technique to probe the atomic ensemble temperature, we confirmed that all motional d.o.f. can be cooled close to the ground state, despite the close proximity of the hot fiber surface. This work constitutes, to our knowledge, the first experimental evidence of manipulation of all motional d.o.f. at the quantum level for atoms coupled to a nanophotonic structure.

Such control is of major importance for cold-atom-based nanophotonic devices, where ground-state cooling ensures the homogeneity of the atom-waveguide coupling. For instance, it could improve the performances of atomic Bragg mirrors [32,33], quantum memories [34,35], or squeezing protocols [36]. The possibility to cool atoms using exclusively guided light fields also opens up opportunities for the design of compact cold-atom-based devices that could be assembled to perform, for instance, precision sensing or quantum networking. Moreover, our technique can be of interest to cool nanofiber-trapped atoms in environments where optical access is restricted, such as in certain cryogenic systems [37].

In a more general context, our results pave the way towards atomic quantum probes for the study of nearsurface effects-for example, the experimental study of optical near-field forces [38-43], self-organization [44-46], or quantum friction [47]. Ground-state cooling also constitutes a well-defined starting point for the loading of atoms in surface-induced potentials [48].

\section{ACKNOWLEDGMENTS}

We thank P. Jessen and C. Clausen for stimulating discussions and helpful comments. Financial support by the European Research Council (CoG NanoQuaNt), the Austrian Science Fund (FWF, SFB NextLite Project No. F 4908-N23 and DK CoQuS Project No. W 1210-N16), the Wiener Wissenschafts- und Technologie Fonds (WWTF) Project No. MA16-066 ("SEQUEX"), and the Austrian Academy of Sciences (ÖAW, ESQ Discovery Grant) is gratefully acknowledged.

\section{APPENDIX: FIT MODEL FOR SPECTRA}

Here, we describe the model we use to fit the spectra in Fig. 4 in order to infer the temperature of the atomic ensemble. A more extensive description of the fluorescence spectrum of a trapped two-level atom can be found, for instance, in the work of Jessen [31]. This model is based on a rate-equation approach and was shown to be consistent with the full-quantum approach presented in Ref. [49]. Here, we only retrace the main steps allowing us to compute the spectrum. For clarity, we restrict our discussion to the one-dimensional case. However, this method can be straightforwardly extended to the three-dimensional case considered in this paper.

We consider an atom in a harmonic trap of frequency $\omega_{x}$, and we denote the trap's eigenenergies and eigenstates as
$E_{n}$ and $|n\rangle$, respectively. This atom is illuminated by a laser field, and we consider the induced Raman scattering between different motional states in the trap. We consider the case of a small saturation parameter, i.e., of low intensity and/or large detunings, so the scattering processes are mostly coherent. Here, $\boldsymbol{k}_{L}$ (resp. $\boldsymbol{k}_{S}$ ) and $\omega_{L}$ (resp. $\omega_{S}$ ) are the wave vector and frequency of the excitation laser (resp. of the scattered photon). The scattering process gives rise to transitions between motional states $n_{i}$ and $n_{f}$ at a rate $\gamma_{n_{i} \rightarrow n_{f}}$, which can be computed using Fermi's golden rule:

$$
\gamma_{n_{i} \rightarrow n_{f}}=\gamma_{0}\left|\left\langle n_{f}\left|e^{i\left(\boldsymbol{k}_{S}-\boldsymbol{k}_{L}\right) \cdot \boldsymbol{r}}\right| n_{i}\right\rangle\right|^{2},
$$

where $\gamma_{0}$ is a global scattering rate depending on the laser's parameters. In the Lamb-Dicke regime, we can perform a series expansion of Eq. (A1) in orders of the small parameter $\eta_{x}=\Delta k_{x} x_{0}$, with $x_{0}$ the size of the harmonic oscillator ground state and $\Delta k_{x}=\left(\boldsymbol{k}_{S}-\boldsymbol{k}_{L}\right) \cdot \boldsymbol{e}_{x}$. For $\eta_{x} \ll 1$, we are only left with the transitions $n \rightarrow n$ and $n \rightarrow n \pm 1$, and we obtain the following rates:

$$
\begin{gathered}
\gamma_{n}^{(0)} \equiv \gamma_{n \rightarrow n}=\gamma_{0}\left[1-(2 n+1) \eta_{x}^{2}\right] \\
\gamma_{n}^{(+)} \equiv \gamma_{n \rightarrow n+1}=\gamma_{0} \eta_{x}^{2}(n+1), \\
\gamma_{n}^{(-)} \equiv \gamma_{n \rightarrow n-1}=\gamma_{0} \eta_{x}^{2} n
\end{gathered}
$$

corresponding to the carrier, and the Stokes and anti-Stokes sidebands, respectively.

Starting from a state $|n\rangle$ with a thermal occupation probability $\pi_{n}$, the transition $n \rightarrow n \pm 1$ gives rise to a spectral contribution centered around a frequency $\omega_{S}=$ $\omega_{L} \mp \omega_{x}$, with an amplitude $A_{n}^{( \pm)}=\pi_{n} \gamma_{n}^{( \pm)}$. We follow the approximation made in Ref. [31] and compute the total spectrum by incoherently summing the contributions of all the $n \rightarrow n \pm 1$ transitions. This method corresponds to considering a set of independent Bloch equations for twolevel systems $\{|n\rangle,|n+1\rangle\}$. Following the calculations from Ref. [31], we infer the width $\Gamma_{n, n+1}$ of the spectral contribution of the $n \rightarrow n+1$ process by computing the mean total departure rate from the states $|n\rangle$ and $|n+1\rangle$, obtained by integrating Eqs. (A3) and (A4) over all possible scattering angles. We are then left with

$$
\Gamma_{n, n+1}=\epsilon \gamma_{0} \eta_{x}^{2}(n+1) \equiv \Gamma_{0}(n+1),
$$

where $\epsilon$ is a geometrical factor coming from the spatial integration and depending on the details of the emission pattern. From Eq. (A5), we notice that the width of the contribution of the $n \rightarrow n+1$ transition increases with $n$. In our fitting routine, we use the width of the $0 \rightarrow 1$ transition, $\Gamma_{0}$, as a free parameter. Finally, the fluorescence spectrum $S(\omega)$ is given by summing the contributions of all $n \rightarrow n \pm 1$ processes. We get 


$$
\begin{aligned}
S(\omega)= & \xi \sum_{n=0}^{+\infty}\left\{\pi_{n} \gamma_{n}^{(+)} \frac{\Gamma_{n, n+1} /(2 \pi)}{\left(\omega+\omega_{x}\right)^{2}+\left(\Gamma_{n, n+1} / 2\right)^{2}}\right. \\
& \left.+\pi_{n+1} \gamma_{n+1}^{(-)} \frac{\Gamma_{n, n+1} /(2 \pi)}{\left(\omega-\omega_{x}\right)^{2}+\left(\Gamma_{n, n+1} / 2\right)^{2}}\right\},
\end{aligned}
$$

where $\xi$ is a global amplitude factor, accounting for the overall detection efficiency. We assume a Lorentzian shape for each spectral contribution. However, the numerical method we use to compute the power spectral density from the SPCM data utilizes a moving time window, resulting in Gauss-shaped peaks. The fits shown in Fig. 4 were hence performed using a Gaussian profile rather than a Lorentzian one. These fits also account for the trap anharmonicity, which can be easily modeled by adding a transition-dependent frequency shift in Eq. (A6). The temperature of the atomic ensemble is included in the distribution of populations $\left\{\pi_{n}\right\}$. For a thermal distribution, we expect $\pi_{n}=(1-q) q^{n}$, with $q=\exp \left[-\hbar \omega_{x} /\left(k_{\mathrm{B}} T_{x}\right)\right]$, where $T_{x}$ is the temperature and $k_{\mathrm{B}}$ Boltzmann's constant. In the fit routine, we use $q$ as a free parameter. The mean number of motional quanta is then given by $\left\langle n_{x}\right\rangle=q /(1-q)$. Note that the temperature can be mainly inferred from the relative power scattered in the Stokes and anti-Stokes sidebands, i.e., from the relative area of the corresponding peaks in the fluorescence spectrum. This makes the measurement of temperature quite robust against some experimental details, such as the exact shape of these peaks. This was confirmed by comparing the results of a fit using Gaussian and Lorentzian peak profiles. Although the Gaussian model fits the data better, both models yield comparable temperature estimates.

[1] A. D. Cronin, J. Schmiedmayer, and D. E. Pritchard, Optics and Interferometry with Atoms and Molecules, Rev. Mod. Phys. 81, 1051 (2009).

[2] A. D. Ludlow, M. M. Boyd, J. Ye, E. Peik, and P. O. Schmidt, Optical Atomic Clocks, Rev. Mod. Phys. 87, 637 (2015).

[3] I. Bloch, J. Dalibard, and S. Nascimbène, Quantum Simulations with Ultracold Quantum Gases, Nat. Phys. 8, 267 (2012).

[4] H. J. Kimble, The Quantum Internet, Nature (London) 453, 1023 (2008).

[5] P. Lodahl, S. Mahmoodian, S. Stobbe, A. Rauschenbeutel, P. Schneeweiss, J. Volz, H. Pichler, and P. Zoller, Chiral Quantum Optics, Nature (London) 541, 473 (2017).

[6] E. Vetsch, D. Reitz, G. Sagué, R. Schmidt, S. T. Dawkins, and A. Rauschenbeutel, Optical Interface Created by LaserCooled Atoms Trapped in the Evanescent Field Surrounding an Optical Nanofiber, Phys. Rev. Lett. 104, 203603 (2010).

[7] J. D. Thompson, T. G. Tiecke, N. P. de Leon, J. Feist, A. V. Akimov, M. Gullans, A. S. Zibrov, V. Vuletic, and
M. D. Lukin, Coupling a Single Trapped Atom to a Nanoscale Optical Cavity, Science 340, 1202 (2013).

[8] I. Bouchoule, H. Perrin, A. Kuhn, M. Morinaga, and C. Salomon, Neutral Atoms Prepared in Fock States of a One-Dimensional Harmonic Potential, Phys. Rev. A 59, R8 (1999).

[9] L. Förster, M. Karski, J.-M. Choi, A. Steffen, W. Alt, D. Meschede, A. Widera, E. Montano, J. H. Lee, W. Rakreungdet, and P. S. Jessen, Microwave Control of Atomic Motion in Optical Lattices, Phys. Rev. Lett. 103, 233001 (2009).

[10] C. Henkel, S. Potting, and M. Wilkens, Loss and Heating of Particles in Small and Noisy Traps, Appl. Phys. B 69, 379 (1999).

[11] J. M. McGuirk, D. M. Harber, J. M. Obrecht, and E. A. Cornell, Alkali-Metal Adsorbate Polarization on Conducting and Insulating Surfaces Probed with Bose-Einstein Condensates, Phys. Rev. A 69, 062905 (2004).

[12] C. Henkel and M. Wilkens, Heating of Trapped Atoms Near Thermal Surfaces, Europhys. Lett. 47, 414 (1999).

[13] C. Wuttke, G. D. Cole, and A. Rauschenbeutel, Optically Active Mechanical Modes of Tapered Optical Fibers, Phys. Rev. A 88, 061801 (2013).

[14] B. Albrecht, Y. Meng, C. Clausen, A. Dareau, P. Schneeweiss, and A. Rauschenbeutel, Fictitious Magnetic-Field Gradients in Optical Microtraps as an Experimental Tool for Interrogating and Manipulating Cold Atoms, Phys. Rev. A 94, 061401 (2016).

[15] C. Østfeldt, J.-B. S. Béguin, F. T. Pedersen, E. S. Polzik, J. H. Müller, and J. Appel, Dipole Force Free Optical Control and Cooling of Nanofiber Trapped Atoms, Opt. Lett. 42, 4315 (2017).

[16] S. E. Hamann, D. L. Haycock, G. Klose, P. H. Pax, I. H. Deutsch, and P. S. Jessen, Resolved-Sideband Raman Cooling to the Ground State of an Optical Lattice, Phys. Rev. Lett. 80, 4149 (1998).

[17] V. Vuletić, C. Chin, A. J. Kerman, and S. Chu, Degenerate Raman Sideband Cooling of Trapped Cesium Atoms at Very High Atomic Densities, Phys. Rev. Lett. 81, 5768 (1998).

[18] A. J. Kerman, V. Vuletić, C. Chin, and S. Chu, Beyond Optical Molasses: 3D Raman Sideband Cooling of Atomic Cesium to High Phase-Space Density, Phys. Rev. Lett. 84, 439 (2000).

[19] M. Gröbner, P. Weinmann, E. Kirilov, and H.-C. Nägerl, Degenerate Raman Sideband Cooling of ${ }^{39} \mathrm{~K}$, Phys. Rev. A 95, 033412 (2017).

[20] J. Hu, A. Urvoy, Z. Vendeiro, V. Crépel, W. Chen, and V. Vuletić, Creation of a Bose-Condensed Gas of ${ }^{87} \mathrm{Rb}$ by Laser Cooling, Science 358, 1078 (2017).

[21] P. S. Jessen, C. Gerz, P. D. Lett, W. D. Phillips, S. L. Rolston, R. J. C. Spreeuw, and C. I. Westbrook, Observation of Quantized Motion of Rb Atoms in an Optical Field, Phys. Rev. Lett. 69, 49 (1992).

[22] F. Le Kien, J. Q. Liang, K. Hakuta, and V. I. Balykin, Field Intensity Distributions and Polarization Orientations in a Vacuum-Clad Subwavelength-Diameter Optical Fiber, Opt. Commun. 242, 445 (2004).

[23] C. Cohen-Tannoudji and J. Dupont-Roc, Experimental Study of Zeeman Light Shifts in Weak Magnetic Fields, Phys. Rev. A 5, 968 (1972). 
[24] R. Mitsch, C. Sayrin, B. Albrecht, P. Schneeweiss, and A. Rauschenbeutel, Exploiting the Local Polarization of Strongly Confined Light for Sub-Micrometer-Resolution Internal State Preparation and Manipulation of Cold Atoms, Phys. Rev. A 89, 063829 (2014).

[25] D. Reitz, C. Sayrin, R. Mitsch, P. Schneeweiss, and A. Rauschenbeutel, Coherence Properties of NanofiberTrapped Cesium Atoms, Phys. Rev. Lett. 110, 243603 (2013).

[26] A. M. Kaufman, B. J. Lester, and C. A. Regal, Cooling a Single Atom in an Optical Tweezer to Its Quantum Ground State, Phys. Rev. X 2, 041014 (2012).

[27] H.-G. Hong, W. Seo, M. Lee, W. Choi, J.-H. Lee, and K. An, Spectral Line-Shape Measurement of an Extremely Weak Amplitude-Fluctuating Light Source by Photon-CountingBased Second-Order Correlation Spectroscopy, Opt. Lett. 31, 3182 (2006).

[28] M. Das, A. Shirasaki, K. P. Nayak, M. Morinaga, F. Le Kien, and K. Hakuta, Measurement of Fluorescence Emission Spectrum of Few Strongly Driven Atoms Using an Optical Nanofiber, Opt. Express 18, 17154 (2010).

[29] M. Lindberg, Resonance Fluorescence of a Laser-Cooled Trapped Ion in the Lamb-Dicke Limit, Phys. Rev. A 34, 3178 (1986).

[30] J. Javanainen and S. Stenholm, Laser Cooling of Trapped Particles III: The Lamb-Dicke Limit, Appl. Phys. A 24, 151 (1981).

[31] P. S. Jessen, Heterodyne Spectroscopy of Resonance Fluorescence, Ph.D. thesis, University of Aarhus, 1993.

[32] N. V. Corzo, B. Gouraud, A. Chandra, A. Goban, A. S. Sheremet, D. V. Kupriyanov, and J. Laurat, Large Bragg Reflection from One-Dimensional Chains of Trapped Atoms Near a Nanoscale Waveguide, Phys. Rev. Lett. 117, 133603 (2016).

[33] H. L. Sørensen, J.-B. Béguin, K. W. Kluge, I. Iakoupov, A. S. Sørensen, J. H. Müller, E. S. Polzik, and J. Appel, Coherent Backscattering of Light off One-Dimensional Atomic Strings, Phys. Rev. Lett. 117, 133604 (2016).

[34] B. Gouraud, D. Maxein, A. Nicolas, O. Morin, and J. Laurat, Demonstration of a Memory for Tightly Guided Light in an Optical Nanofiber, Phys. Rev. Lett. 114, 180503 (2015).

[35] C. Sayrin, C. Clausen, B. Albrecht, P. Schneeweiss, and A. Rauschenbeutel, Storage of Fiber-Guided Light in a Nanofiber-Trapped Ensemble of Cold Atoms, Optica 2, 353 (2015).
[36] X. Qi, B. Q. Baragiola, P. S. Jessen, and I. H. Deutsch, Dispersive Response of Atoms Trapped Near the Surface of an Optical Nanofiber with Applications to Quantum Nondemolition Measurement and Spin Squeezing, Phys. Rev. A 93, 023817 (2016).

[37] M. Hafezi, Z. Kim, S. L. Rolston, L. A. Orozco, B. L. Lev, and J. M. Taylor, Atomic Interface between Microwave and Optical Photons, Phys. Rev. A 85, 020302 (2012).

[38] S. Scheel, S. Y. Buhmann, C. Clausen, and P. Schneeweiss, Directional Spontaneous Emission and Lateral CasimirPolder Force on an Atom Close to a Nanofiber, Phys. Rev. A 92, 043819 (2015).

[39] S. Sukhov, V. Kajorndejnukul, and A. Dogariu, Dynamic Consequences of Optical Spin-Orbit Interaction, Nat. Photonics 9, 809 (2015).

[40] F. J. Rodríguez-Fortuño, N. Engheta, A. Martínez, and A. V. Zayats, Lateral Forces on Circularly Polarizable Particles Near a Surface, Nat. Commun. 6, 8799 (2015).

[41] F. Kalhor, T. Thundat, and Z. Jacob, Universal SpinMomentum Locked Optical Forces, Appl. Phys. Lett. 108, 061102 (2016).

[42] M. Antognozzi et al., Direct Measurements of the Extraordinary Optical Momentum and Transverse Spin-Dependent Force Using a Nano-Cantilever, Nat. Phys. 12, 731 (2016).

[43] K. Y. Bliokh, A. Y. Bekshaev, and F. Nori, Extraordinary Momentum and Spin in Evanescent Waves, Nat. Commun. 5, 3300 (2014).

[44] D. E. Chang, J. I. Cirac, and H. J. Kimble, Self-Organization of Atoms along a Nanophotonic Waveguide, Phys. Rev. Lett. 110, 113606 (2013).

[45] T. Grießer and H. Ritsch, Light-Induced Crystallization of Cold Atoms in a 1D Optical Trap, Phys. Rev. Lett. 111, 055702 (2013).

[46] Z. Eldredge, P. Solano, D. Chang, and A. V Gorshkov, SelfOrganization of Atoms Coupled to a Chiral Reservoir, Phys. Rev. A 94, 053855 (2016).

[47] F. Intravaia, V. E Mkrtchian, S. Y. Buhmann, S. Scheel, D. A. R. Dalvit, and C. Henkel, Friction Forces on Atoms after Acceleration, J. Phys. Condens. Matter 27, 214020 (2015).

[48] D. E. Chang, K. Sinha, J. M. Taylor, and H. J. Kimble, Trapping Atoms Using Nanoscale Quantum Vacuum Forces, Nat. Commun. 5, 4343 (2014).

[49] J. I. Cirac, R. Blatt, A. S. Parkins, and P. Zoller, Spectrum of Resonance Fluorescence from a Single Trapped Ion, Phys. Rev. A 48, 2169 (1993). 